\title{
The Bologna motor and non-motor prospective study on parkinsonism at onset (BoProPark): study design and population
}

\author{
Giovanna Calandra-Buonaura $^{1,2} \cdot$ Luisa Sambati $^{1,2} \cdot$ Francesca Baschieri $^{1,2} \cdot$ Maria Vitiello $^{1,2} \cdot$ Manuela Contin ${ }^{1,2}$. \\ Caterina Tonon $^{2,3} \cdot$ Sabina Capellari ${ }^{1,2} \cdot$ Federica Provini $^{1,2} \cdot$ Pietro Cortelli $^{1,2}$ (1) on behalf of the BoProPark Study \\ Group
}

Received: 7 December 2019 / Accepted: 20 February 2020 / Published online: 26 March 2020

(C) The Author(s) 2020

\begin{abstract}
Objective The Bologna motor and non-motor prospective study on parkinsonism at onset (BoProPark) was designed to prospectively characterize motor and non-motor features in patients with a progressive neurodegenerative disease starting with parkinsonism since early disease stage and to investigate their diagnostic and prognostic role in the differential diagnosis of Parkinson's disease from atypical parkinsonisms. The aim of this paper is to describe the method and population of the BoProPark study.

Methods Patients referred to our Department with parkinsonism within 3 years from motor onset were recruited. Secondary causes of parkinsonism were excluded. Each patient underwent a comprehensive evaluation of motor and non-motor symptoms, assessed by means of quantitative, objective instrumental tests in addition to scales and questionnaires. The evaluations were performed at enrolment (T0), after 16 months (T1) and after 5 years (T2). Diagnoses were made according to consensus criteria. Results We recruited 150 patients, with mean age $61.5 \pm 9.8$ years and mean disease duration $20 \pm 9$ months. H\&Y stage was 1 in $47.3 \%$ and 2 in $46.7 \%$ of cases. Mean UPDRS-III was $17.7 \pm 9.2$. Fifty-four patients were on dopaminergic treatment with median levodopa equivalent daily dose (LEDD) of $200 \mathrm{mg}$.

Conclusions We expect that the prospective nature of the BoProPark study as well as the comprehensive, instrumental evaluation of motor and non-motor symptoms in patients with parkinsonism will provide important new insights for both clinical practice and research. Our data could be used for comparison with other cohorts and shared with national and international collaborators to develop new innovative projects.
\end{abstract}

Keywords Parkinson's disease · Atypical parkinsonisms $\cdot$ Cohort study $\cdot$ Study design $\cdot$ Motor symptoms $\cdot$ Non-motor symptoms

\begin{tabular}{llll}
\multicolumn{2}{l}{ Abbreviations } & uAP & Unspecified atypical parkinsonism \\
PD & Parkinson's disease & $N$ & Number of patients \\
MSA & Multiple system atrophy & UPDRS-III & Unified PD Rating Scale - part III \\
DLB & Dementia with Lewy bodies & LEDD & Levodopa equivalent daily dose \\
PSP & Progressive supranuclear palsy & & \\
CBS & Corticobasal syndrome & &
\end{tabular}

\section{Introduction}

Pietro Cortelli

pietro.cortelli@unibo.it

1 IRCCS Istituto delle Scienze Neurologiche di Bologna, Via Altura 3, 40139 Bologna, Italy

2 Dipartimento di Scienze Biomediche e Neuromotorie, Università di Bologna, Bologna, Italy

3 Diagnostica Funzionale Neuroradiologica, IRCCS Istituto delle Scienze Neurologiche di Bologna, Bologna, Italy
Parkinsonism is defined by the presence of a combination of bradykinesia, rigidity, tremor, and postural instability. It may be due to secondary causes (e.g., vascular encephalopathy, drugs' side effects) or may occur in the context of neurodegenerative disorders, such as Parkinson's disease (PD) or atypical parkinsonisms (APs). PD is the second most common neurodegenerative disease, carrying a high burden in terms of disability and healthcare cost worldwide [1]. APs are distinct 
entities, which at early disease stage could mimic PD [2], but during the disease course develop motor or non-motor features that exclude or are atypical for PD. Moreover, APs are characterized by a worse prognosis and different therapeutic needs compared to PD.

Nowadays, with the better understanding of the pathogenesis of these conditions and the aim of the development of disease-modifying therapies [3-5], the need for finding suitable clinical and instrumental markers to confirm diagnosis and track progression has become of crucial importance. Several prospective studies have been promoted for this purpose, focusing on frequency, severity, impact on diagnosis, and prognosis of motor as well as non-motor symptoms (e.g., autonomic failure, sleep disorders, cognitive impairment). However, most of these studies mainly assessed nonmotor symptoms by means of questionnaires and scales that, while being comprehensive and standardized screening tools, do not allow objective quantification and only capture patient self-reported symptoms [6]. Moreover, the majority of these studies targeted patients with a single diagnosis, even though differentiating PD from APs at onset is still challenging [7].

The Bologna motor and non-motor prospective study on parkinsonism at onset (BoProPark) was designed to prospectively characterize motor and non-motor features, assessed by means of quantitative, objective instrumental tests in addition to scales and questionnaires, in patients with a progressive neurodegenerative disease starting with parkinsonism since early disease stage and to investigate their diagnostic and prognostic role in the differential diagnosis of these diseases.

The aim of this paper is to describe the method and population of the BoProPark study.

\section{Materials and methods}

The BoProPark is a prospective, observational, single-center study.

We enrolled all consecutive patients aged $18-80$ years presenting with parkinsonism (tremor, bradykinesia, rigidity, postural instability) with a progressive course and within 3 years from motor onset that were referred from September 2007 up to November 2018 to the Movement Disorders Clinic of the Department of Biomedical and Neuromotor Sciences, University of Bologna. The neurodegenerative origin of the parkinsonism was confirmed in all patients by pathological single-photon emission computerized tomography for imaging the dopamine transporter (SPECT DaTSCAN). Secondary causes of parkinsonism were excluded before enrollment by means of appropriate investigations including brain magnetic resonance imaging (MRI). Other exclusion criteria to enter the study were concurrent clinically severe medical or psychiatric disease that could have interfered with study results.
The study was initially supported by the strategic research program "Bando ricerca finalizzata 2006" of the Italian Ministry of Health (reference number RFPS2006-7-336374) and subsequently continued as independent research and approved by the Ethics Committee of the Local Health Authority of Bologna (reference number 09070). The study was performed in accordance with the Declaration of Helsinki.

All participants gave their written informed consent to participate in the study.

\section{BoProPark protocol}

According to the BoProPark study, each patient underwent the same protocol (Table 1) at baseline (T0), after 16 months (T1) and 5 years (T2). The time window between $\mathrm{T} 0$ and $\mathrm{T} 1$ evaluations was chosen in order to get information on the early evolution of the disease, i.e., within the first 5 years, when correct clinical diagnosis is more challenging [29]. The second follow-up (T2) was set at reasonable time to collect data on an established clinical diagnosis, considering that PD and APs have different progression and prognosis. Moreover, because inclusion criteria requires that patients have parkinsonism within 3 years from motor onset, this time frame allowed us to evaluate most of the APs over their disease course, taking into account that survival in this cases is usually less than 10 years.

Drug naive patients at T0 started levodopa plus dopadecarboxylase inhibitor (carbidopa or benserazide) treatment titrated in 1 month up to $200+50 \mathrm{mg} /$ die.

The protocol included the following evaluations:

(a) History taking: age, sex, occupation, family history, past medical history, drug history and concomitant medications, age at disease (parkinsonism) onset, disease duration, characteristics of onset and progression, symptoms at initial presentation, occurrence of motor and nonmotor symptoms (history consistent with bradykinesia, rigidity, tremor, postural instability, other additional movement disorders such as dystonia or myoclonus and their body distribution, falls, dysphagia, dysarthria, dysphonia, higher mental functions, depression, sleep disturbances, autonomic symptoms including symptoms suggestive of orthostatic hypotension, urinary urgency, frequency, incontinence, incomplete bladder emptying, sexual dysfunction, sweating abnormalities, constipation, diarrhea, vision disturbances); severity, timing and latency from disease onset were recorded for each symptom and sign; dopaminergic treatment.

(b) Body mass index.

(c) Neurological examination.

(d) Quantification of motor impairment and disease severity by means of Unified PD Rating Scale - part III (UPDRSIII) and Hoehn \& Yahr (H\&Y) stage $[8,9]$. 
Table 1 The BoProPark protocol

\begin{tabular}{ll}
\hline General clinical assessment & -History taking \\
& -Complete neurological examination \\
Motor assessment & -Unified PD Rating Scale - part III [8] \\
& -Hoehn \& Yahr stage [9] \\
& -Subacute challenge test with levodopa [10] \\
& -Cardiovascular reflex tests [11, 12] \\
Autonomic system & -Scales for Outcomes in Parkinson's Disease - Autonomic questionnaire [13] \\
& -Whole-night video-polysomnography [14] \\
Sleep & -Parkinson's disease sleep scale 1 [15] \\
& -REM sleep behavior disorder questionnaire [16] \\
& -Bologna questionnaire on sleepiness-related symptoms [17] \\
& -Restless Legs Syndrome criteria according to the International \\
& Classification of Sleep Disorders [18] and International \\
& Restless Legs Syndrome Study Group rating scale [19, 20] \\
& -Epworth sleepiness scale [21] \\
& -PD quality of life questionnaire [22] \\
& -Mini-Mental State Examination [23] \\
& -Simple Copy Design Test [24] \\
& -Selective Visual Attention Test (Stroop Test) [25] \\
-Phonemic and Semantic Verbal Fluency Test [26] & -Brief Mental Deterioration Battery [24] \\
Cuality of life & -Beck's Depression Inventory [27] \\
Cognitive and behavioral & -State-Trait Anxiety Inventory [28] \\
\hline &
\end{tabular}

(e) Patients treated with levodopa underwent quantification of motor response to levodopa through a subacute challenge test with a standard oral dose of levodopa plus carbidopa or benserazide $(100+25 \mathrm{mg})$ based on a kinetic-dynamic approach [10]. Drug naïve patients at T0 underwent this examination within 6 months after the end of levodopa titration. Patient's motor response was objectively assessed before and after standard intervals from drug dose with finger tapping performed on a computer-based system; simultaneous blood venous samples were withdrawn for measuring levodopa plasma concentration; dyskinesias, when present, were also rated at the same times as motor responses by the Clinical Dyskinesia Rating Scale [30]; blood pressure in supine position and after 3 min of standing was measured before and $1 \mathrm{~h}$ after drug administration to detect possible levodopa-induced or worsened orthostatic hypotension.

(f) Evaluation of autonomic control of the cardiovascular system through cardiovascular reflex tests performed according to standardized procedures with continuous monitoring of beat-to-beat blood pressure, heart rate, oronasal airflow, abdominal breathing, and peripheral vasomotor tone and video: tilt test $\left(65^{\circ}\right.$ for $\left.10 \mathrm{~min}\right)$, Valsalva's maneuver (forced expiratory pressure of $40 \mathrm{mmHg}$ for $15 \mathrm{~s}$ ), deep breathing test ( 6 breaths/min), cold face test (cold stimulus on forehead for $1 \mathrm{~min}$ ), handgrip test (1/3 of maximal effort for $5 \mathrm{~min}$ ) [11, 12]; blood pressure and heart rate changes from baseline in response to these maneuvers were used to calculate indices of sympathetic and parasympathetic activity and baroreflex integrity and to detect the presence of orthostatic hypotension.

(g) Presence of symptoms of autonomic dysfunction through the questionnaire Scales for Outcomes in Parkinson's Disease - Autonomic (SCOPA-Aut) [13].

(h) Sleep study by means of whole-night videopolysomnography: electroencephalogram (C3-A2, O2A1, Cz-A1), right and left electrooculogram, surface electromyogram from submental, intercostalis, right and left extensor carpi radialis and tibialis anterior muscles, tracheal microphone, oronasal airflow, thoracic and abdominal respirogram, electrocardiogram, oxyhemoglobin saturation by means of finger oximeter and synchronized video recording [14].

(i) Sleep questionnaires and scales: PD sleep scale 1 [15], REM sleep behavior disorder (RBD) questionnaire [16], Bologna questionnaire on sleepiness-related symptoms [17], Restless Legs Syndrome criteria according to the International Classification of Sleep Disorders (ICSD-3) [18] and International Restless Legs Syndrome Study Group rating scale $[19,20]$, and Epworth sleepiness scale [21].

(j) Evaluation of quality of life by means of 39-item PD questionnaire [22]. 
(k) Cognitive and behavioral comprehensive assessment evaluating global cognition, verbal and visual memory, attention, executive and visuospatial function, language, depression, and anxiety. Neuropsychological evaluation included the following tests corrected for age, sex, and education according to Italian standardizations: Mini-Mental State Examination [23], Simple Copy Design Test [24], Selective Visual Attention Test (Stroop Test) [25], Phonemic and Semantic Verbal Fluency Test [26], and the Brief Mental Deterioration Battery, consisting of Rey's Auditory Verbal Learning Test (immediate and delayed recall) [24], Visual Search Test (Barrage test) [24], Immediate Visual Memory Test [24], and Simple Verbal Analogies Test [31]. The Battery outcomes is a measure of global cognitive functioning, called Final Result [32, 33]. All patients also filled the 21-items Beck's Depression Inventory [27] and the State-Trait Anxiety Inventory [28].

Demographic, clinical, and instrumental data were collected using a standard and anonymous form and entered in an ad hoc database. This database was specifically developed in Microsoft.net framework by SparkBio Srl (Bologna, Italy) for the purpose of collecting and storing data of this study in a comprehensive, standard, and safe way and making subsequent statistical analysis more straightforward. Moreover, this database allowed us to identify potential missing or erroneous information that were immediately pursued to guarantee the completeness and accuracy of the data. Importantly, the database was also implemented with a deterministic algorithm based on international criteria for PD [34], Parkinson' disease with dementia (PDD) [35, 36], multiple system atrophy (MSA) [37], dementia with Lewy bodies (DLB) [38], progressive supranuclear palsy (PSP) [39], and corticobasal syndrome (CBS) [40] that checked the information and automatically provided a diagnosis that was recorded in the database itself and available for comparison and confirmation at future visits. In this regard, the database was designed as a questionnaire for all relevant diagnostic information needed to make a diagnosis according to the aforementioned criteria. Patients not fulfilling any diagnostic criteria were diagnosed as unspecified atypical parkinsonism (uAP). All diagnoses were independently confirmed by three neurologists expert in movement disorders who were blinded to the diagnosis provided by the database. Diagnoses made at baseline (T0) were revisited at $\mathrm{T} 1$ and $\mathrm{T} 2$ based on the results obtained from these follow-up evaluations.

Results of tests and questionnaires were recorded in ad hoc databases for statistical analysis.

Patients were also followed up at our department according to clinical practice and were regularly assessed for routine visits every 6-12 months as needed.

\section{Statistical analysis}

In the present article, we performed a descriptive analysis using IBM SPSS Statistics software (version 25). The distribution of continuous variables was confirmed by visual analysis of the Q-Q plots and the Shapiro Wilk test. Continuous variables were expressed using mean and standard deviation while categorical variables using frequencies and proportions.

\section{Results}

A total of 150 patients were recruited. Demographics and clinical characteristics at $\mathrm{T} 0$ are reported in Table 2. Mean age was $61.5 \pm 9.8$ years, and mean disease duration was 20 \pm 9 months. H\&Y stage was 1 in $47.3 \%$ and 2 in $46.7 \%$ of cases. Mean UPDRS III was $17.7 \pm 9.2$. Fifty-four patients were on dopaminergic treatment with median levodopa equivalent daily dose (LEDD) of $200 \mathrm{mg}$ (36-700). The following diagnoses were made at T0: 108 PD, 4 MSA, 2 DLB, 3 PSP, 2 CBS, and $31 \mathrm{uAP}$ (Fig. 1).

\section{Discussion}

The BoProPark study aims to describe and evaluate the diagnostic and prognostic role of motor and non-motor symptoms in a cohort of patients with a neurodegenerative disease starting with parkinsonism within 3 years from motor onset and prospectively followed up to reach

Table 2 Demographics and clinical characteristics of patients at T0

\begin{tabular}{ll}
\hline$N$ & 150 \\
Males & $78(52 \%)$ \\
Age (years) & $61.5 \pm 9.8(37-83)$ \\
Disease duration (months) & $20 \pm 9(2-36)$ \\
Hoehn \& Yahr stage & \\
1 & $71(47.3 \%)$ \\
2 & $70(46.7 \%)$ \\
3 and 4 & $9(6 \%)$ \\
UPDRS-III & $17.7 \pm 9.2(5-51)$ \\
LEDD (mg)* & $200(36-700)$ \\
Diagnosis & \\
PD & $108(72 \%)$ \\
MSA & $4(2.7 \%)$ \\
DLB & $2(1.3 \%)$ \\
PSP & $3(2 \%)$ \\
CBS & $2(1.3 \%)$ \\
uAP & $31(20.7 \%)$ \\
\hline
\end{tabular}

Data are expressed as number (percentage) or mean \pm standard deviation (min - max)

*Data expressed as median ( $\min -\max )$ 
Fig. 1 Study flowchart, $\mathrm{PD}=$ Parkinson's disease; $\mathrm{MSA}=$ multiple system atrophy; DLB = dementia with Lewy bodies; PSP = progressive supranuclear palsy; CBS = corticobasal syndrome; $\mathrm{uAP}=$ unspecified atypical parkinsonism. (*) all uAP (according to data available). (**) $6 \mathrm{PD}$ and $2 \mathrm{uAP}$ (according to data available)

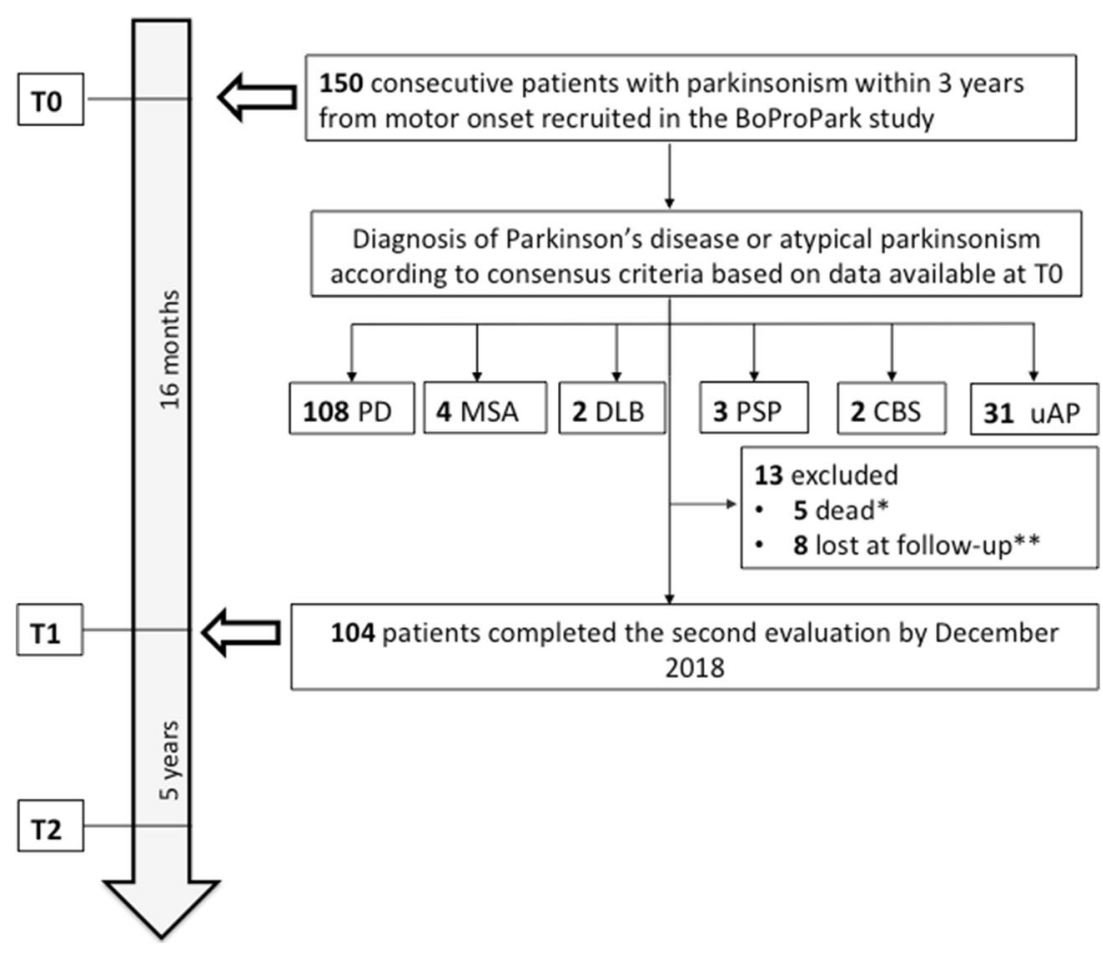

a more robust clinical diagnosis. Motor and non-motor symptoms were evaluated by means of clinical and instrumental assessments according to standardized procedures. According to the results of clinical and instrumental assessments, diagnoses were revisited and confirmed at each evaluation (T0, T1, and T2) and at further follow-up.

In this paper, we describe study method and characteristics of patients at enrollment. Our cohort is representative of parkinsonian patients at disease onset referring to a tertiary Movement Disorder Center in Italy and Europe [41]. By further analyzing baseline and followup data, we expect to find specific clinical and instrumental markers for each domain assessed that can be helpful to the clinician in the diagnostic work-up and management of patient with parkinsonisms. Furthermore, our data could be used for comparison with other cohorts [6] and shared with national and international collaborators to develop new innovative projects. In addition, we could identify different patient populations with specific clinical characteristics that could be suitable for future studies assessing, for example, new and emerging MRI approaches like quantitative susceptibility mapping that might provide additional useful information for the early detection of PD and APs. We believe that our study will have important implications for clinical practice as well as in the research field.

Acknowledgments This work is dedicated to professor Pasquale Montagna.
BoProPark Study Group collaborators Giorgio Barletta ${ }^{1,2}$ (autonomic nervous system laboratory), Giuseppe Caltabiano ${ }^{1,2}$ (sleep laboratory), Annagrazia Cecere ${ }^{1,2}$ (autonomic nervous system laboratory), Roberto Gallassi (neuropsychology), Giulia Giannini ${ }^{1,2}$ (patients' recruitment), Pietro Guaraldi ${ }^{1,3}$ (patients' recruitment), Raffaele Lodi ${ }^{2,4}$ (neuroimaging), Giovanna Lopane ${ }^{1}$ (pharmacology), David Neil Manners ${ }^{4}$ (neuroimaging), Paolo Martinelli ${ }^{1}$ (patients' recruitment), Filomena Miele ${ }^{1,2}$ (sleep laboratory), Francesco Mignani ${ }^{1,2}$ (sleep laboratory), Susan Mohamed $^{1}$ (pharmacology), Stefania Nassetti ${ }^{1}$ (patients' recruitment), Federico Oppi ${ }^{1}$ (neuropsychology), Piero Parchi ${ }^{1,2}$ (neuropathology), Giulia Pierangeli ${ }^{1,2}$ (autonomic nervous system laboratory), Roberto Poda $^{1}$ (neuropsychology), Cesa Scaglione ${ }^{1}$ (patients' recruitment), Laura Solieri $^{1,2}$ (autonomic nervous system laboratory), Michelangelo Stanzani-Maserati ${ }^{1}$ (neuropsychology), Claudia Testa ${ }^{4}$ (neuroimaging)

${ }^{1}$ IRCCS Istituto delle Scienze Neurologiche di Bologna, Ospedale Bellaria, via Altura 3, 40138 Bologna, Italy

${ }^{2}$ Dipartimento di Scienze Biomediche e Neuromotorie, Università di Bologna, Bologna, Italy

${ }^{3}$ Neurology Outpatient Clinic, Department of Primary Care, Local Health Authority of Modena, Italy

${ }^{4}$ IRCCS Istituto delle Scienze Neurologiche di Bologna, Diagnostica Funzionale Neuroradiologica, Bologna, Italy

Funding information The study was supported by the strategic research program "Bando ricerca finalizzata 2006" of the Italian Ministry of Health (reference number RFPS2006-7-336374) and was co-financed with contributions from "5 x 1000" - support for neuroscience health research 2015.

\section{Compliance with ethical standards}

Conflict of interest The authors declare that they have no conflict of interest. 
Ethical approval Ethics Committee of the Local Health Authority 113 of Bologna (reference number 09070).

Open Access This article is licensed under a Creative Commons Attribution 4.0 International License, which permits use, sharing, adaptation, distribution and reproduction in any medium or format, as long as you give appropriate credit to the original author(s) and the source, provide a link to the Creative Commons licence, and indicate if changes were made. The images or other third party material in this article are included in the article's Creative Commons licence, unless indicated otherwise in a credit line to the material. If material is not included in the article's Creative Commons licence and your intended use is not permitted by statutory regulation or exceeds the permitted use, you will need to obtain permission directly from the copyright holder. To view a copy of this licence, visit http://creativecommons.org/licenses/by/4.0/.

\section{References}

1. Ray Dorsey E, Elbaz A, Nichols E et al (2018) Global, regional, and national burden of Parkinson's disease, 1990-2016: a systematic analysis for the global burden of disease study 2016. Lancet Neurol 17:939-953. https://doi.org/10.1016/S1474-4422(18) 30295-3

2. Ali K, Morris HR (2015) Parkinson's disease: chameleons and mimics. Pract Neurol 15:14-25. https://doi.org/10.1136/ practneurol-2014-000849

3. Elkouzi A, Vedam-Mai V, Eisinger RS, Okun MS (2019) Emerging therapies in Parkinson disease - repurposed drugs and new approaches. Nat Rev Neurol 15:204-223. https://doi.org/10.1038/ s41582-019-0155-7

4. Boxer AL, Yu JT, Golbe LI, Litvan I, Lang AE, Höglinger GU (2017) Advances in progressive supranuclear palsy: new diagnostic criteria, biomarkers, and therapeutic approaches. Lancet Neurol 16: 552-563. https://doi.org/10.1016/S1474-4422(17)30157-6

5. Lopez-Cuina M, Foubert-Samier A, Tison F, Meissner WG (2018) Present and future of disease-modifying therapies in multiple system atrophy. Auton Neurosci Basic Clin 211:31-38. https://doi.org/ 10.1016/j.autneu.2017.12.008

6. Heinzel S, Lerche S, Maetzler W, Berg D (2017) Global, yet incomplete overview of cohort studies in Parkinson's disease. J Park Dis 7:423-432. https://doi.org/10.3233/JPD-171100

7. Hughes AJ, Daniel SE, Ben-Shlomo Y, Lees AJ (2002) The accuracy of diagnosis of parkinsonian syndromes in a specialist movement disorder service. Brain 125:861-870. https://doi.org/10.1093/ brain/awf080

8. Fahn S, Elton R, Members of the UPDRS Development Committee (1987) Unified Parkinson's disease rating scale. In: Fahn S, Marsden C, Calne D, Goldstein M (eds) Recent developments in Parkinson's disease, vol 153-63. Macmillan Health Care Information, Florham Park, pp 293-304

9. Hoehn MM, Yahr MD (1967) Parkinsonism: onset, progression, and mortality. Neurology 17:427-442. https://doi.org/10.1212/ WNL.17.5.427

10. Contin M, Riva R, Martinelli P, Albani F, Avoni P, Baruzzi A (2001) Levodopa therapy monitoring in patients with Parkinson disease: a kinetic-dynamic approach. Ther Drug Monit 23:621629. https://doi.org/10.1097/00007691-200112000-00005

11. Corazza I, Barletta G, Guaraldi P, Cecere A, Calandra-Buonaura G, Altini E, Zannoli R, Cortelli P (2014) A new integrated instrumental approach to autonomic nervous system assessment. Comput Methods Prog Biomed 117:267-276. https://doi.org/10.1016/j. cmpb.2014.08.002
12. Ewing D (1978) Cardiovascular reflexes and autonomic neuropathy. Clin Sci Mol Med 55:321-327

13. Visser M, Marinus J, Stiggelbout AM, Van Hilten JJ (2004) Assessment of autonomic dysfunction in Parkinson's disease: the SCOPA-AUT. Mov Disord 19:1306-1312. https://doi.org/10.1002/ mds. 20153

14. Vetrugno R, Provini F, Cortelli P, Plazzi G, Lotti EM, Pierangeli G, Canali C, Montagna P (2004) Sleep disorders in multiple system atrophy: a correlative video-polysomnographic study. Sleep Med 5: 21-30. https://doi.org/10.1016/j.sleep.2003.07.002

15. Chaudhuri KR, Pal S, DiMarco A, Whately-Smith C, Bridgman K, Mathew R, Pezzela FR, Forbes A, Högl B, Trenkwalder C (2002) The Parkinson's disease sleep scale: a new instrument for assessing sleep and nocturnal disability in Parkinson's disease. J Neurol Neurosurg Psychiatry 73:629-635. https://doi.org/10.1136/jnnp. 73.6 .629

16. Scaglione C, Vignatelli L, Plazzi G, Marchese R, Negrotti A, Rizzo G, Lopane G, Bassein L, Maestri M, Bernardini S, Martinelli P, Abbruzzese G, Calzetti S, Bonuccelli U, Provini F, Coccagna G, Bologna, Genova, Parma and Pisa Universities group for the study of REM Sleep Behavior Disorder in Parkinson's Disease (2005) REM sleep behaviour disorder in Parkinson's disease: a questionnaire-based study. Neurol Sci 25:316-321. https://doi.org/ 10.1007/s10072-004-0364-7

17. Rinaldi R, Vignatelli L, D'Alessandro R et al (2001) Validation of symptoms related to excessive daytime sleepiness. Neuroepidemiology 20:248-256

18. American Academy of Sleep Medicine (2014) International classification of sleep disorders, 3rd ed. Darien

19. Walters AS (1995) Toward a better definition of the restless legs syndrome. The International Restless Legs Syndrome Study Group. Mov Disord 10:634-642. https://doi.org/10.1002/mds.870100517

20. Walters AS, LeBrocq C, Dhar A et al (2003) Validation of the International Restless Legs Syndrome Study Group rating scale for restless legs syndrome. Sleep Med 4:121-132. https://doi.org/ 10.1016/S1389-9457(02)00258-7

21. Vignatelli L, Plazzi G, Barbato A et al (2003) Italian version of the Epworth sleepiness scale: external validity. Neurol Sci 23:295-300. https://doi.org/10.1007/s100720300004

22. Peto V, Jenkinson C, Fitzpatrick R (1998) PDQ-39: a review of the development, validation and application of a Parkinson's disease quality of life questionnaire and its associated measures. J Neurol 245:S10-S14. https://doi.org/10.1007/PL00007730

23. Measso G, Cavarzeran F, Zappalà G et al (1993) The mini-mental state examination: normative study of an Italian random sample. Dev Neuropsychol 9:77-85. https://doi.org/10.1080/ 87565649109540545

24. Carlesimo GA, Caltagirone C, Gainotti G et al (1996) The mental deterioration battery: normative data, diagnostic reliability and qualitative analyses of cognitive impairment. Eur Neurol 36:378384. https://doi.org/10.1159/000117297

25. Caffarra P, Vezzadini G, Dieci F et al (2002) Una versione abbreviata del test di Stroop: dati normativi nella popolazione italiana. Nuova Riv di Neurol 12:111-115

26. Novelli G, Papagno C, Capitani E et al (1986) Tre test clinici di ricerca e produzione lessicale. Taratura su sogetti normali. Arch Psicol Neurol Psichiatr 47:477-506

27. Beck AT, Ward CH, Mendelson M et al (1961) An inventory for measuring depression. Arch Gen Psychiatry 4:561-571. https://doi. org/10.1001/archpsyc.1961.01710120031004

28. Spielberger CD (1987) State-trait anxiety inventory. Anxiety 19: 2009

29. Adler CH, Beach TG, Hentz JG, Shill HA, Caviness JN, DriverDunckley E, Sabbagh MN, Sue LI, Jacobson SA, Belden CM, Dugger BN (2014) Low clinical diagnostic accuracy of early vs 
advanced Parkinson disease: clinicopathologic study. Neurology 83:406-412. https://doi.org/10.1212/WNL.0000000000000641

30. Hagell P, Widner H (1999) Clinical rating of dyskinesias in Parkinson's disease: use and reliability of a new rating scale. Mov Disord 14:448-455. https://doi.org/10.1002/1531-8257(199905) 14:3<448::AID-MDS1010>3.0.CO;2-0

31. Gallassi R, Sambati L, Stanzani Maserati M, Poda R, Oppi F, de Matteis M, Marano G (2014) Simple verbal analogies test: normative data on a short task exploring abstract thinking. Aging Clin Exp Res 26:67-71. https://doi.org/10.1007/s40520-013-0180-0

32. Gallassi R, Lenzi P, Stracciari A, Lorusso S, Ciardulli C, Morreale A, Mussuto V (1986) Neuropsychological assessment of mental deterioration: purpose of a brief battery and a probabilistic definition of "normality" and "non-normality.". Acta Psychiatr Scand 74: 62-67. https://doi.org/10.1111/j.1600-0447.1986.tb06228.x

33. Gallassi R, Morreale A, Di Sarro R, Lorusso S (2002) Value of clinical data and neuropsychological measures in probable Alzheimer's disease. Arch Gerontol Geriatr 34:123-134. https:// doi.org/10.1016/S0167-4943(01)00204-7

34. Gelb DJ, Oliver E, Gilman S (1999) Diagnostic criteria for Parkinson disease. Arch Neurol 56:33-39. https://doi.org/10. 1001/archneur.56.1.33

35. Emre M, Aarsland D, Brown R et al (2007) Clinical diagnostic criteria for dementia associated with Parkinson's disease. Mov Disord 22:1689-1707

36. Dubois B, Burn D, Goetz C et al (2007) Diagnostic procedures for Parkinson's disease dementia: recommendations from the Movement Disorder Society Task Force. Mov Disord 22:2314 2324. https://doi.org/10.1002/mds.21844

37. Gilman S, Wenning GK, Low PA, Brooks DJ, Mathias CJ, Trojanowski JQ, Wood NW, Colosimo C, Dürr A, Fowler CJ, Kaufmann H, Klockgether T, Lees A, Poewe W, Quinn N,
Revesz T, Robertson D, Sandroni P, Seppi K, Vidailhet M (2008) Second consensus statement on the diagnosis of multiple system atrophy. Neurology 71:670-676. https://doi.org/10.1212/01.wnl. 0000324625.00404 .15

38. McKeith IG, Dickson DW, Lowe J, Emre M, O'Brien JT, Feldman H, Cummings J, Duda JE, Lippa C, Perry EK, Aarsland D, Arai H, Ballard CG, Boeve B, Burn DJ, Costa D, del Ser T, Dubois B, Galasko D, Gauthier S, Goetz CG, Gomez-Tortosa E, Halliday G, Hansen LA, Hardy J, Iwatsubo T, Kalaria RN, Kaufer D, Kenny RA, Korczyn A, Kosaka K, Lee VM, Lees A, Litvan I, Londos E, Lopez OL, Minoshima S, Mizuno Y, Molina JA, MukaetovaLadinska EB, Pasquier F, Perry RH, Schulz JB, Trojanowski JQ, Yamada M, Consortium on DLB (2005) Diagnosis and management of dementia with Lewy bodies: third report of the DLB consortium. Neurology 65:1863-1872

39. Litvan I, Agid Y, Calne D, Campbell G, Dubois B, Duvoisin RC, Goetz CG, Golbe LI, Grafman J, Growdon JH, Hallett M, Jankovic J, Quinn NP, Tolosa E, Zee DS (1996) Clinical research criteria for the diagnosis of progressive supranuclear palsy (Steele-RichardsonOlszewski syndrome): report of the NINDS-SPSP international workshop. Neurology 47:1-9. https://doi.org/10.1212/WNL.47.1.1

40. Riley DE, Lang AE (2000) Clinical diagnostic criteria. In: Litvan I, Goetz CG, Lang AE (eds) Corticobasal degeneration and related disorders. Lippincott Williams \& Wilkins, Philadelphia, pp 29-39

41. Baldereschi M, Di Carlo A, Rocca WA et al (2000) Parkinson's disease and parkinsonism in a longitudinal study: two-fold higher incidence in men. Neurology 55:1358-1363. https://doi.org/10. 1212/WNL.55.9.1358

Publisher's note Springer Nature remains neutral with regard to jurisdictional claims in published maps and institutional affiliations. 